\title{
Particle re-acceleration in the ICM and low-frequency observations
}

\author{
Gianfranco Brunetti \\ INAF - Istituto di Radioastronomia, via P. Gobetti 101, I-40129 Bologna, Italy \\ email: brunetti@ira.inaf.it
}

\begin{abstract}
The particle reaceleration model is one of the most promising possibilities to explain the Mpc-scale diffuse radio emission detected in a number of galaxy clusters. Ongoing and future radio observations at low frequencies may help in constraining and testing this model.
\end{abstract}

Keywords. acceleration of particles, turbulence, galaxies: clusters: general

The Mpc-scale radio non-thermal emission (Radio Halos, RHs) detected in a growing number of galaxy clusters (GCs) proves the presence of $\mathrm{GeV}$ radiating electrons (e.g., Feretti 2005). Relativistic hadrons should accumulate in GCs and direct measurements of the hadronic content may come from future gamma-ray observations (e.g., Blasi 2004).

The origin of the radio emitting electrons in RHs is still a matter of debate: these particles have a lifetime much shorter than that necessary to diffuse over Mpc scales. This diffusion problem (Jaffe 1977) poses a fundamental theoretical problem on the origin of the emitting particles. They may be re-accelerated or they may be secondary products of collisions between CR protons and thermal protons in the ICM (e.g., Sarazin 2004).

The injection and confinement of CR hadrons in the ICM has been discussed in several papers (Völk et al. 1996; Berezinsky et al. 1997; Ensslin et al. 1997). These particles actually accumulate in GCs for periods comparable to the age of the clusters. Also, the amplification of the magnetic field in GCs and its decay take cosmological time scales (e.g., Dolag 2006; Subramanian 2006). Thus, in the framework of the secondary model, RHs should be long-lived sources, with lifetimes $\sim$ the ages of clusters. On the other hand, these sources are always found in merging clusters (e.g., Buote 2001) and are quite rare, at least at the level of present radio observations (e.g., Giovannini et al. 1999), which means that their typical lifetime is probably relatively short ( $1 \mathrm{Gyr}$, Hwang 2004$)$.

A major step forward can be achieved by looking at non-radio emitting clusters; the steep spectrum of RHs makes low frequency observations efficient tools in this research. A pointed survey of a sample of about 50 massive GCs in the redshift bin $z=0.2-0.4$ has been recently carried out at the GMRT at $604 \mathrm{MHz}$ (Venturi et al. 2006, 2007). Only about $15 \%$ of these massive GCs host giant RHs, while no diffuse radio emission is detected in the great majority of these GCs at the brightness level of the observations which is $\sim 10$ times below that of the NVSS at $1.4 \mathrm{GHz}$. Although the radio power $\left(P_{\mathrm{R}}\right)$ of giant RHs is known to correlate with the X-ray luminosity $\left(L_{\mathrm{X}}\right)$ of the parent GCs (e.g., Feretti 2005), these results suggest that the bulk of GCs does not follow this correlation. The region in the $P_{\mathrm{R}}-L_{\mathrm{X}}$ plane that is filled by the bulk of the massive GCs in the GMRT survey is indeed found to lie about 2 orders of magnitude below (in terms of radio power) that spanned by the $P_{\mathrm{R}}-L_{\mathrm{X}}$ correlation (Brunetti et al., in prep). These findings have important consequences on our present view of the origin of RHs in GCs. They confirm that RHs are transient phenomena while the bulk of GCs is basically non-radio emitting, and this disfavours any scenario in which the emitting electrons are injected in GCs for cosmological (long) time-scales. 
Apparently the reacceleration model nicely fits the evidence that $\mathrm{RHs}$ are transient and always found in dynamically disturbed GCs. If stochastic reacceleration is driven by turbulence generated in cluster mergers, the cascading time of turbulence from large scales $(300-500 \mathrm{kpc})$ sets a natural timescale for the lifetime of RHs which is of the order of 1 Gyr (or even less). This model (e.g., Brunetti et al. 2001; Petrosian 2001) is based on the complex issue of stochastic particle re-acceleration whose physical details are still poorly understood and difficult to test. Theoretical attempts to calculate the time-dependent stochastic particle acceleration process in the ICM usually focus on the Alfvén modes (e.g., Brunetti et al. 2004), and assume that these modes are injected at small, resonant, scales by some process connected to the presence of turbulence at larger scales. An additional possibility comes from the action of compressible modes injected at large scales in GCs during cluster-cluster mergers. Fast modes and magnetosonic waves couple with relativistic particles via TTD-resonance and non-resonant turbulent compression, and this might provide a contribution to the reacceleration process (Brunetti 2006; Brunetti \& Lazarian, in prep).

Attempts to calculate the statistical properties of RHs in the framework of the reacceleration model have been carried out only recently (e.g., Cassano \& Brunetti 2005). The probability to develop a $\mathrm{RH}$ is found to depend critically on the mass of the hosting GC as the injection of turbulence on Mpc scales is more efficient with increasing cluster mass. This means that there is a threshold in cluster mass below which giant RHs rarely develop, and this drives a low radio power cut-off in the expected luminosity functions of giant RHs (Cassano et al. 2006). On the other hand, this mass threshold should depend on observing frequency: at lower frequencies the synchrotron emission comes from lower energy electrons which can be accelerated much easily, and thus RHs in GCs with slightly lower mass come into play. Since the number of GCs per comoving volume increases with decreasing mass, the number of detectable giant RHs in the re-acceleration model is expected to increase at lower observing frequencies. This is a clear expectation of the model which can be tested by future low frequency observations: present calculations show that LOFAR and the LWA might discover more than 1000 new giant RHs (Cassano et al. 2006).

\section{References}

Blasi, P. 2004, J. Korean Astron. Soc. 37, 483

Berezinsky, V. S., Blasi, P., \& Ptuskin, V. S. 1997, ApJ, 487, 529

Brunetti, G. 2006, AN, 327, 615

Brunetti, G., Setti, G., Feretti, L., \& Giovannini, G. 2001, MNRAS, 320, 365

Brunetti, G., Blasi, P., Cassano, R., \& Gabici, S. 2004, MNRAS, 350, 1174

Buote, D. A. 2001, ApJ (Letters), 553, L15

Cassano, R., \& Brunetti, G. 2005, MNRAS, 357, 1313

Cassano, R., Brunetti, G., \& Setti, G. 2006, MNRAS, 369, 1577

Dolag, K. 2006, AN, 327, 575

Ensslin, T. A., Biermann, P. L., Kronberg, P. P., \& Wu, X-P. 1997, ApJ, 477, 560

Feretti, L. 2005, Adv. Space Res., 36, 729

Giovannini, G., Tordi, M., \& Feretti, L. 1999, New Astron., 4, 141

Hwang, C.-Y. 2004, J. Korean Astron. Soc., 37, 461

Jaffe, W. J. 1977, ApJ, 212, 1

Petrosian, V. 2001, ApJ, 557, 560

Sarazin, C. 2004, J. Korean Astron. Soc., 37, 433

Subramanian, K., Shukurov, A., \& Haugen, N. E. L. 2006, MNRAS, 366, 1437

Venturi, T., Parma, P., de Ruiter, H. R. 2006, MemSAI Suppl., 10, 111

Venturi, T., Giacintucci, S., Brunetti, et al. 2007, A\&A, 463, 937

Völk, H. J., Aharonian F. A., \& Breitschwerdt D. 1996, Space Sci. Revs., 75, 279 